\title{
Noncollinear cluster magnetism in the framework of the Hubbard model
}

\author{
Miguel A. Ojeda and J. Dorantes-Dávila \\ Instituto de Física, Universidad Autónoma de San Luis Potosí, \\ Alvaro Obregón 64, 78000 San Luis Potosí, Mexico \\ G. M. Pastor \\ Laboratoire de Physique Quantique, Unité Mixte de Recherche 5626 du CNRS, \\ Université Paul Sabatier, 118 route de Narbonne, F-31062 Toulouse, France
}

(February 22, 2018)

\begin{abstract}
Noncollinear magnetic states in clusters are studied by using the single-band Hubbard Hamiltonian. The unrestricted Hartree-Fock (UHF) approximation is considered without imposing any symmetry constraints neither to the size or orientation of the local magnetic moments $\left\langle\vec{S}_{l}\right\rangle$ nor to the local charge densities $\left\langle n_{l}\right\rangle$. A variety of qualitatively different selfconsistent solutions is obtained as a function of cluster size, structure, number of valence electrons $\nu$ and Coulomb interaction strength $U / t$. This includes inhomogeneous density distributions, paramagnetic solutions, magnetic solutions with collinear moments and noncollinear spin arrangements that show complex antiferromagnetic and ferromagnetic-like orders. The environment dependence of the magnetic properties is analyzed giving emphasis to the effects of antiferromagnetic frustrations in compact structures close to halfband filling. Electron correlation effects are quantified by comparing UHF and exact results for the local magnetic moments, total spin, spin-correlation functions and structural stability of 13-atom clusters. Goals and limitations of the present noncollinear approach are discussed.
\end{abstract}

PACS numbers: 36.40.Cg, 71.24.+q, 75.10.Lp

\section{INTRODUCTION}

Clusters may show specific phenomena that do not have an equivalent in the thermodynamic limit and that are therefore of interest in both basic and applied science. Moreover, the evolution of their physical properties with increasing cluster size provides new perspectives for understanding the microscopic origin of condensed-matter properties [1]. In this context, the study of magnetism in metals posses a particularly interesting and challenging problem since the properties of magnetic metals often change dramatically as the electrons of an isolated atom become part of a cluster of several atoms and delocalize. It is therefore of fundamental importance to understand how itinerant magnetism, as found for example in $3 d$ transition-metal (TM) solids, develops starting from localized atomic magnetic moments. The strong dependence of the magnetic behavior as a function of system size, structure and composition opens in addition the possibility of using clusters to tailor new magnetic materials for specific technological purposes. Consequently, the relation between local atomic environment, cluster structure and magnetism has also implications of practical interest.

Most theoretical studies of itinerant cluster magnetism have been performed using Hartree-Fock (HF) and local spin density (LSD) methods [2]. Exact many-body calculations are presently limited to simple models, such as the Hubbard model, and to systems containing a small number of sites [3 5]. Within mean-field approximations (HF or LSD) the self-consistent spin polarizations are usually restricted to be collinear, i.e., the direction of all local magnetic moments is assumed to be the same. However, the well known sensitivity of itinerant magnetism to the local environment of the atoms [2 suggests that other instabilities towards spiral-like spin-density-waves (SDW's) [6] or even more complex magnetic structures should also be possible in general. For example, the tendency to antiferromagnetic (AF) order close to half-band filling and the presence of triangular loops in compact structures generates magnetic frustrations that may easily yield complex arrangements of the local magnetic moments. Moreover, the reduction of local coordination numbers at the surface of clusters removes constraints between the local moments and could favor the development of low-symmetry spin polarizations. In solids, noncollinear magnetic structures have been identified experimentally and theoretically already for a long time [6 9]. In contrast, very little is known at present in the case of finite systems 10 12]. Fully unrestricted $a b$ initio calculations of noncollinear spin arrangements have been performed only recently for very small $\mathrm{Fe}_{N}$ clusters [12]. The investigation of magnetic phenomena of this kind requires a symmetry unrestricted approach in which no a priori assumptions are made concerning the relative orientation of the local magnetic moments, thereby enlarging the number of degrees of freedom of the problem.

The main purpose of this paper is to investigate the characteristics of noncollinear magnetic states in finite clusters. We consider the single-band Hubbard model and determine the ground-state magnetic properties in a fully unrestricted Hartree-Fock approximation. The 
theoretical approach, outlined in Sec. II is applied to clusters having $N \leq 43$ atoms. The results presented in Sec. III analyze noncollinear magnetic behaviors for a few representative compact structures. Several examples are given that illustrate the large variety of 3dimensional magnetic arrangements obtained in the selfconsistent calculations as a function of the Coulomb repulsion strength $U / t$, band filling $\nu / N$ and cluster structure. In Sec. [V] we compare the UHF results with exact diagonalization calculations for $N \leq 13$ atoms (Lanczos method) [5]. The role of quantum fluctuations beyond mean field and the consequences of the often artificial breaking of spin-symmetry implied by the formation of the noncollinear local moments are discussed. Taking into account that approximations such as UHF are unavoidable for larger clusters and for more realistic model Hamiltonians, it is of considerable interest to test the validity of these methods in order to improve the interpretation of the approximate results and to obtain a more accurate description of magnetic phenomena in clusters.

\section{THEORETICAL METHOD}

The single-band Hubbard Hamiltonian 13 is given by

$$
H=-t \sum_{\langle l, m\rangle, \sigma} \hat{c}_{l \sigma}^{\dagger} \hat{c}_{m \sigma}+U \sum_{l} \hat{n}_{l \uparrow} \hat{n}_{l \downarrow}
$$

where $\hat{c}_{l \sigma}^{\dagger}\left(\hat{c}_{l \sigma}\right)$ is the creation (annihilation) operator of an electron at site $l$ with spin $\sigma$, and $\hat{n}_{l \sigma}=\hat{c}_{l \sigma}^{\dagger} \hat{c}_{l \sigma}$ is the corresponding number operator $\left(\hat{n}_{l}=\sum_{\sigma} \hat{n}_{l \sigma}\right)$. The first sum runs over all pairs of nearest neighbors (NN) and the second over all sites. The model is characterized by the dimensionless parameter $U / t$, that measures the relative importance between kinetic and Coulomb energies, by the cluster structure, that defines the kinetic energy operator, and by the number of valence electrons $\nu$. The variations of $U / t$ can be associated to a uniform relaxation of the interatomic distances (e.g., $t \propto R_{i j}^{-5}$ for TM's) or to changes in the spatial extension of the atomic-like wave function, as in different elements within the same group. Different $\nu$ 's correspond to different band-fillings $\nu / N$ that may be associated qualitatively to the variations of $\nu / N$ across a TM $d$ series. In spite of its simplicity, this Hamiltonian has played, together with related models, a major role in guiding our understanding of the many-body properties of metals and of lowdimensional magnetism. It is the purpose of this work to use it to investigate the properties of noncollinear itinerant magnetism in small compact clusters.

In the unrestricted Hartree-Fock (UHF) approximation the ground state for $\nu$ electrons is a single Slater determinant that can be written as

$$
|\mathrm{UHF}\rangle=\left[\prod_{k}^{\nu} \hat{a}_{k}^{\dagger}\right]|\mathrm{vac}\rangle
$$

The single-particle states

$$
\hat{a}_{k}^{\dagger}=\sum_{l, \sigma=\uparrow, \downarrow} A_{l \sigma}^{k} \hat{c}_{l \sigma}^{\dagger}
$$

are linear combinations of the atomic-like orbitals associated to $\hat{c}_{l \sigma}^{\dagger}$. Notice that in Eq. 2.3 we allow for the most general superposition of single-electron states since $\hat{a}_{k}^{\dagger}$ may involve a mixture of both $\uparrow$ and $\downarrow$ spin components. The eventually complex coefficients $A_{i \sigma}^{k}$ are determined by minimizing the energy expectation value $E_{\mathrm{UHF}}=\langle\mathrm{UHF}|H| \mathrm{UHF}\rangle$. In terms of the density matrix

$$
\rho_{l \sigma, m \sigma^{\prime}} \equiv\left\langle\mathrm{UHF}\left|\hat{c}_{l \sigma}^{\dagger} \hat{c}_{m \sigma^{\prime}}\right| \mathrm{UHF}\right\rangle=\sum_{k=1}^{\nu} \bar{A}_{l \sigma}^{k} A_{m \sigma^{\prime}}^{k},
$$

this is given by

$$
E_{\mathrm{UHF}}=-t \sum_{\langle l, m\rangle, \sigma} \rho_{l \sigma, m \sigma}+U \sum_{l}\left(\rho_{l \uparrow, l \uparrow} \rho_{l \downarrow, l \downarrow}-\left|\rho_{l \uparrow, l \downarrow}\right|^{2}\right) .
$$

The energy minimization and the normalization constraints on the wave function lead to the usual selfconsistent equations

$$
-t \sum_{m} A_{m \sigma}^{k}+U\left(A_{l \sigma}^{k} \rho_{l \bar{\sigma}, l \bar{\sigma}}-A_{l \bar{\sigma}}^{k} \rho_{l \bar{\sigma}, l \sigma}\right)=\varepsilon_{k} A_{l \sigma}^{k} .
$$

For a given solution, the average local electronic density $\left\langle n_{l}\right\rangle$ is given by

$$
\left\langle n_{l}\right\rangle=\rho_{l \uparrow, l \uparrow}+\rho_{l \downarrow, l \downarrow},
$$

and the spin polarizations vectors $\left\langle\vec{S}_{l}\right\rangle=$ $\left(\left\langle S_{l}^{x}\right\rangle,\left\langle S_{l}^{y}\right\rangle,\left\langle S_{l}^{z}\right\rangle\right)$ by

$$
\begin{aligned}
& \left\langle S_{l}^{x}\right\rangle=\left(\rho_{l \uparrow, l \downarrow}+\rho_{l \downarrow, l \uparrow}\right) / 2, \\
& \left\langle S_{l}^{y}\right\rangle=-i\left(\rho_{l \uparrow, l \downarrow}-\rho_{l \downarrow, l \uparrow}\right) / 2, \\
& \left\langle S_{l}^{z}\right\rangle=\left(\rho_{l \uparrow, l \uparrow}-\rho_{l \downarrow}, l \downarrow\right) / 2 \text {. }
\end{aligned}
$$

The usual collinear UHF approach is recovered when $\rho_{l \sigma, l \bar{\sigma}}=0 \forall l$, i.e., when all magnetic moments $\left\langle\vec{S}_{l}\right\rangle$ are parallel to $z$. In practice, several random spin arrangements are used as starting points of the selfconsistent procedure in order to ensure that the final result corresponds to the true UHF ground state. In case of multiple selfconsistent solutions (nonequivalent by rotations) the UHF energies are compared. $E_{\mathrm{UHF}}$ can be rewritten as

$$
E_{\mathrm{UHF}}=-t \sum_{\langle l, m\rangle, \sigma} \rho_{l \sigma, m \sigma}+\frac{U}{4} \sum_{l}\left\langle n_{l}\right\rangle^{2}-U \sum_{l}\left|\left\langle\vec{S}_{l}\right\rangle\right|^{2} .
$$

One observes that the Hartree-Fock Coulomb energy $E_{C}^{H F}$ - the sum of the 2nd and 3rd terms in Eq. 2.9) 
- favors a uniform density distribution and the formation of local moments $\left\langle\vec{S}_{l}\right\rangle$. Due to the local character of Hubbard's Coulomb interaction, the relative orientation of the different $\left\langle\vec{S}_{l}\right\rangle$ does not affect $E_{C}^{H F}$. It is therefore the optimization of the kinetic energy that eventually leads to the formation of complex magnetic structures with $\left|\left\langle\vec{S}_{l}\right\rangle \cdot\left\langle\vec{S}_{m}\right\rangle\right| \neq 1$ or to non-uniform density distributions $\left\langle n_{l}\right\rangle$.

As a result of the tendency to avoid double orbital occupancies, the UHF solutions often correspond to states of broken symmetry: spin-density waves (SDW's), charge density waves (CDW's) or both. The spin-rotational invariance of Eqs. (2.2 2.6) implies that the energy is unchanged after a rotation of the whole spin arrangement $\left\{\left\langle\vec{S}_{l}\right\rangle, l=1, \ldots, N\right\}$. Therefore, if $\left\langle\vec{S}_{l}\right\rangle \neq 0$ one has a set of linearly independent congruent solutions $|\mathrm{UHF} k\rangle$ $(k \geq 2)$ which have the same average energy $E_{\mathrm{UHF}}$ and which differ from each other only by the orientation of the spin polarizations relative to the cluster structure. The illustrations of spin arrangements shown in Sec. III correspond to one of these SDW's, which is chosen only for the sake of clarity. The set of UHF solutions may be used to restore the symmetry appropriate to the exact ground state $\left|\Psi_{0}\right\rangle$ thereby improving the approximate wave function. For instance, for some SDW's having $\sum_{l}\left\langle\vec{S}_{l}\right\rangle=0$ one may consider the spin-symmetrized Hartree-Fock (SSHF) wave function 14, 15]

$$
|\mathrm{SSHF}\rangle=\frac{|\mathrm{UHF} 1\rangle+|\mathrm{UHF} 2\rangle}{\sqrt{2(1+\langle\mathrm{UHF} 1 \mid \mathrm{UHF} 2\rangle)}},
$$

where $|\mathrm{UHF} 2\rangle$ is obtained form $|\mathrm{UHF} 1\rangle$ by interchanging up and down spins. In spite of its simplicity, $|\mathrm{SSHF}\rangle$ goes beyond the UHF approximation and corresponds to a correlated state satisfying

$$
\begin{aligned}
\left|\left\langle\mathrm{SSHF} \mid \Psi_{0}\right\rangle\right| & =\sqrt{2 /(1+\langle\mathrm{UHF} 1 \mid \mathrm{UHF} 2\rangle)}\left|\left\langle\mathrm{UHF} 1 \mid \Psi_{0}\right\rangle\right| \\
& \geq\left|\left\langle\mathrm{UHF} 1 \mid \Psi_{0}\right\rangle\right|=\left|\left\langle\mathrm{UHF} 2 \mid \Psi_{0}\right\rangle\right|
\end{aligned}
$$

If quantum fluctuations between the two SDW's $|\mathrm{UHF} 1\rangle$ and $|\mathrm{UHF} 2\rangle$ are non-negligible ( $\langle\mathrm{UHF} 1|H| \mathrm{UHF} 2\rangle \neq$ $\left.\langle\mathrm{UHF} 1 \mid \mathrm{UHF} 2\rangle E_{\mathrm{UHF}}\right)$ an energy reduction relative to $E_{\mathrm{UHF}}$ is obtained since

$$
E_{\mathrm{SSHF}}=\frac{E_{\mathrm{UHF}}+\langle\mathrm{UHF} 1|H| \mathrm{UHF} 2\rangle}{1+\langle\mathrm{UHF} 1 \mid \mathrm{UHF} 2\rangle} .
$$

$\Delta E=E_{\mathrm{UHF}}-E_{\mathrm{SSHF}}$ measures the importance of these quantum spin fluctuations. More complex symmetrized states involving a linear combination of several degenerate UHF states may be constructed analogously.

In order to quantify the role of electron correlations and to assess goal and limitations of the UHF approximation in applications to finite clusters, we shall also compare some of our results with those obtained by applying exact diagonalization methods [3 5]. In this case the Hubbard model is solved numerically by expanding its ground-state $\left|\Psi_{0}\right\rangle$ in a complete set of basis states
$\left|\Phi_{m}\right\rangle$ which have definite occupation numbers $n_{l \sigma}^{m}$ at all orbitals $l \sigma\left(\hat{n}_{l \sigma}\left|\Phi_{m}\right\rangle=n_{l \sigma}^{m}\left|\Phi_{m}\right\rangle\right.$, with $\left.n_{l \sigma}^{m}=0,1\right)$. $\left|\Psi_{0}\right\rangle$ is written as

$$
\left|\Psi_{0}\right\rangle=\sum_{m} \alpha_{l m}\left|\Phi_{m}\right\rangle
$$

where

$$
\left|\Phi_{m}\right\rangle=\left[\prod_{l \sigma}\left(\hat{c}_{l \sigma}^{\dagger}\right)^{n_{l \sigma}^{m}}\right]|v a c\rangle .
$$

The values of $n_{l \sigma}^{m}$ satisfy the usual conservation of the number of electrons $\nu=\nu_{\uparrow}+\nu_{\downarrow}$ and of the $z$ component of the total spin $S_{z}=\left(\nu_{\uparrow}-\nu_{\downarrow}\right) / 2$, where $\nu_{\sigma}=\sum_{l} n_{l \sigma}^{m}$. Taking into account all possible electronic configurations may imply a considerable numerical effort which depends on the number of atoms and on band filling. For not too large clusters, the expansion coefficients $\alpha_{l m}$ can be determined by sparse-matrix diagonalization procedures. The results presented in this work were obtained using a Lanczos iterative method [4, 5,16$]$. In order to calculate $\left|\Psi_{0}\right\rangle$ one usually works in the subspace of minimal $S_{z}$ since this ensures that there are no a priori restrictions on the total spin $S$. The ground-state spin $S$ is then obtained by applying to $\left|\Psi_{0}\right\rangle$ the total spin operator

$$
\hat{S}^{2}=\sum_{l m} \vec{S}_{l} \cdot \vec{S}_{m}=\sum_{l m}\left[\frac{1}{2}\left(\hat{S}_{l}^{+} \hat{S}_{m}^{-}+\hat{S}_{l}^{-} \hat{S}_{m}^{+}\right)+\hat{S}_{l}^{z} \hat{S}_{m}^{z}\right]
$$

where $\hat{S}_{l}^{+}=\hat{c}_{l \uparrow}^{\dagger} \hat{c}_{l \downarrow}, \hat{S}_{l}^{-}=\hat{c}_{l \downarrow}^{\dagger} \hat{c}_{l \uparrow}$ and $\hat{S}_{l}^{z}=\left(\hat{n}_{l \uparrow}-\hat{n}_{l \downarrow}\right) / 2$. From the expectation values of $\vec{S}_{l} \cdot \vec{S}_{m}$ one also obtains the local magnetic moments $(l=m)$ and the intersite spin correlation functions $(l \neq m)$.

\section{NONCOLLINEAR MAGNETIC ORDER IN COMPACT CLUSTERS}

In Fig. 1 UHF results are given for the local magnetic moments $\mu_{l}=\left|\left\langle\vec{S}_{l}\right\rangle\right|$ and the total magnetic moment $\mu_{T}=\left|\sum_{l}\left\langle\vec{S}_{l}\right\rangle\right|$ of fcc-like clusters having $N=13,19$ and 43 atoms at half band filling $(\nu=N)$. These clusters are formed by adding to a central atom $(l=1)$ the successive shells of its first NN's $(N=13)$, second NN's $(N=19)$ and third NN's $(N=43)$. Several common properties are observed as a function of $U / t$. Starting from the uncorrelated limit $(U=0)$, the total moment $\mu_{T}$ remains approximately constant for $U / t \leq 3-4$. In the weakly interacting regime, the local moments $\mu_{l}$ do not depend strongly on $U / t$ and are generally small. Notice that for $N=43, \mu_{T}$ is not minimal at small $U\left(\mu_{T}=3 / 2\right)$ due to degeneracies in the single-particle spectrum. For larger $U / t, \mu_{T}$ decreases rapidly eventually with some discontinuities and a few oscillations reaching values close to $\mu_{T}=0$ for $U / t \simeq 5-6(U / t \simeq 9$ for $N=43)$. At this 
intermediate range of $U / t$, the $\mu_{l}$ increase more rapidly reaching values not far from saturation at the $U / t$ for which $\mu_{T}$ is minimum $\left(\mu_{l} \simeq 0.40-0.45\right)$. The opposite trends shown by $\mu_{l}$ and $\mu_{T}$ are a clear indication of the expected onset of strong AF-like order at half-band filling. We shall see in the following that this corresponds in fact to noncollinear spin arrangements. If $U / t$ is increased beyond $U / t=5-6$ (beyond $U / t=9$ for $N=43$ ) the local moments do not vary significantly, and $\mu_{T}$ either does not change very much $(N=19)$ or increases monotonically ( $N=13$ and 43 ) remaining always smaller than $1 / 2$.

The changes in $\mu_{l}$ and $\mu_{T}$ are the result of qualitative changes in the magnetic order. As an example we show in Fig. 2 the selfconsistent spin arrangements obtained in fcc clusters with $N=13$ atoms for representative values of $U / t(\nu=N)$ [17]. For small $U / t(U / t<3.7)$ one finds a collinear AF order with small $\mu_{l}$ [Fig. 2(a)]. Here we observe a charge and spin density-wave at the cluster surface that is related to degeneracies in the single-particle spectrum at $U=0$. The atoms belonging to the central (001) plane [shown in grey in Fig. 2(a)] have much larger moments than the atoms at the upper and lower (001) planes. For example for $U / t=0.5, \mu_{l}=0.26 \mu_{\mathrm{B}}$ at the the central plane, while the other surface moments are $\mu_{l}=0.0046 \mu_{\mathrm{B}}$ (see Fig. 1). The central atom has a small spin polarization $\left(\mu_{1}=0.002 \mu_{\mathrm{B}}\right.$ for $\left.U / t=0.5\right)$. Notice that the magnetic order within the upper and lower (001) planes is ferromagnetic-like (with small $\mu_{l}$ ) and that the surface moments belonging to successive (001) planes couple antiferromagnetically. Thus, the magnetic moments at the surface of the central plane are not frustrated since all its NN's are antiparallel to them. This explains qualitatively the larger $\mu_{l}$ found at this plane. In contrast, unavoidable frustrations are found for the smallest magnetic moments at the central site and at the atoms of the upper and lower (001) planes [see Fig. 2(a)]

The crossover from the small- $U / t$ to the large- $U / t$ regime takes place as a succession of noncollinear spin arrangements which attempt to minimize the magnetic frustrations among the increasing local moments. A representative example is shown in Fig. 2(b). While in this case the spin arrangement is noncollinear, all the spin moments still lie in the same plane. The very small values of $\mu_{T}$ shown in Fig. 1 f for intermediate $U / t$ indicate that there is an almost complete cancellation among the $\left\langle\vec{S}_{l}\right\rangle$. However, this type of spin arrangements are quite unstable if the strength of Coulomb interactions is further increased. At $U / t \simeq 5.1$ the cluster adopts a fully 3 dimensional spin structure which remains essentially unchanged even in the strongly correlated limit [Fig. 2(c)]. The spin arrangement can be viewed as a slight distortion of the spin ordering that minimizes the energy of a classical AF Heisenberg model on the surface shell, i.e., ignoring the interactions with the central site. In fact, if the central atom were removed or if it carried no local moment, as it is the case for $\nu=12$, the surface moments $\left\langle\vec{S}_{l}\right\rangle$ would point along the medians of one of the triangles at the surface and would lie all within a plane. The magnetic interactions with the central spin $\left\langle\vec{S}_{1}\right\rangle$ in the 13-atom cluster induce a small tilt $\left\langle S_{l}^{z}\right\rangle$ of the surface spin polarizations $\left\langle\vec{S}_{l}\right\rangle$ which is opposite to $\left\langle\vec{S}_{1}\right\rangle$ $\left(\left\langle\vec{S}_{1}\right\rangle \| \hat{z}\right.$ for $\left.\nu=N=13\right)$. The $\left\langle S_{l}^{z}\right\rangle$ component is the same for all surface sites and depends moderately on $U / t$ $\left(\left|\left\langle S_{l}^{z}\right\rangle\right|=0.023-0.056\right.$ for $\left.U / t \geq 5.1\right)$.

Similar magnetic structures are also found in larger symmetric fcc clusters. In Fig. 3 the self-consistent spin configuration for $N=43$ and $U / t=10$ is illustrated $(\nu=N)$. The moment $\left\langle\vec{S}_{1}\right\rangle$ of the central site points along the (111) direction ( $\perp$ to the plane of Fig. 3] [17]. The other $\left\langle\vec{S}_{m}\right\rangle$ lie almost in the plane of the figure with only small components $\left\langle S_{m}^{z}\right\rangle$ along the (111) direction. As for $N=13,\left\langle S_{m}^{z}\right\rangle$ is induced by the interactions with $\left\langle\vec{S}_{1}\right\rangle$. In fact, if $\left\langle\vec{S}_{1}\right\rangle$ vanished, all the spins would be in the plane of the figure. $\left\langle S_{m}^{z}\right\rangle$ is the same for all atoms in a given $\mathrm{NN}$ shell, and changes sign as we move from the center to the surface of the cluster. For example for $U / t=10, \cos \theta_{1 m}=-0.22$ for $m$ belonging to the first shell, $\cos \theta_{1 m}=0.25$ for $m$ in the second shell and $\cos \theta_{1 m}=0.01$ for $m$ in the third shell $\left(\cos \theta_{l m}=\left\langle\vec{S}_{l}\right\rangle \cdot\left\langle\vec{S}_{m}\right\rangle /\left|\left\langle\vec{S}_{l}\right\rangle\right|\left|\left\langle\vec{S}_{m}\right\rangle\right|\right)$. Notice that the spin ordering of the innermost 13 atoms is similar to the one found in fcc clusters with $N=13$ [Fig. 2(c)]. While these trends hold for symmetric fcc clusters, it is worth to remark that strong modifications of the magnetic order generally occur if the symmetry of the cluster is lowered. For instance, for $N=14$ - an atom added to the closedshell 13-atom cluster - one obtains a ferromagnetic-like coupling between the central atom and some of the first neighbors $(\nu=N$ and $U / t=10)$.

A more detailed account of the spin correlations in the UHF ground state of fcc clusters is provided by the expectation values $\left\langle\vec{S}_{l} \cdot \vec{S}_{m}\right\rangle$. In Fig. 国 the average of $\left\langle\vec{S}_{l} \cdot \vec{S}_{m}\right\rangle$ between NN atoms at different shells is shown. In most cases we observe that $\left\langle\vec{S}_{l} \cdot \vec{S}_{m}\right\rangle<0$ (AF correlations) and that $\left|\left\langle\vec{S}_{l} \cdot \vec{S}_{m}\right\rangle\right|$ increases with increasing $U / t$. A particularly important increase of $\mathrm{AF}$ correlations is observed for intermediate $U / t$ when the local moments $\mu_{l}$ are formed $(U / t \simeq 4.5-5.5$ for $N=13$ and 19, see also Fig. 1). This is consistent with the already discussed decrease of $\mu_{T}$ and the onset of AF order. There are, however, two exceptions to this trend. The correlations $\gamma_{01}$ between the central site and the surface shell for $N=13$ increase somewhat for $U / t \simeq 5\left(\gamma_{01}<0\right)$ implying that these AF correlations first tend to be less important when the $\mu_{l}$ increase. It seems that, as a result of frustrations, the more important increase of AF correlations $\gamma_{11}$ among the surface NN's is done at the expense of the correlations between the central atom and the surface. A similar behavior is found in exact calculations, as it will be shown in Sec. IV. Another interesting case concerns the correlations $\gamma_{12}$ between the first and second atomic shells for $N=19$ and 43 . Here we observe that $\gamma_{12}$ changes sign 
upon going from $N=19$ to $N=43(U / t>5)$. Moreover, once the local moments are formed, $\gamma_{01}$ decreases for $N=19\left[\gamma_{01}(19)<0\right]$ and increases for $N=43$ as $U / t$ increases $\left[\gamma_{01}(43)>0\right]$. This behavior can be qualitatively understood by comparing the surfaces of these clusters. For $N=19$ the outermost spins (shell 2) are free to couple antiferromagnetically with their NN's (shell 1). However, the presence of an additional atomic shell for $N=43$, which has NN's belonging to both the first and second shells, forces a parallel alignment of the spins in shells 1 and 2 in order to allow AF coupling with the third shell. In fact, as shown in Fig. 目(c), $\gamma_{13}$ and $\gamma_{23}$ are the strongest AF correlations in the 43-atom cluster. The same conclusions are drawn by comparing the relative orientations of the spin polarizations (Fig. 3). In particular we observe that the angles $\theta_{23}$ between NN spin polarizations at shells 2 and 3 are the largest in average $\left(\theta_{23}=\pi\right.$ or close to it).

In addition to the AF half-filled case it is also interesting to discuss other band fillings, for example $\nu=N+1$ that is known to develop a FM ground state in the limit of large $U / t$ [18]. In Fig. 5 results are given for $\mu_{l}$ and $\mu_{T}$ in an fcc 13-atom cluster with $\nu=14$ electrons. In this case we observe an essentially monotonic increase of $\mu_{T}$ that reflects the progressive development of a fully polarized ferromagnetic state. Close to the threshold for the onset of ferromagnetism $(4.5 \leq U / t \leq 6.5)$ the changes of the AF-like spin arrangement produce small oscillations of $\mu_{T}$ (see Fig 5). The approximately steplike behavior resembles at first sight the results obtained in collinear mean-field calculations [19]. However, in the present case the physical picture behind the formation of a FM state is quite different. The increase of $\mu_{T}$ close to the steps involves a succession of noncollinear spin arrangements with increasing degree of parallel moment alignment. Moreover, notice that the local moments increase much more rapidly than $\mu_{T}$ approaching saturation $\left(\mu_{l} \simeq 0.40-0.45\right)$ for values of $U / t$ at which $\mu_{T}$ is still small. Thus, the increase of $\mu_{T}$ with $U / t$ is the result of the parallel alignment of already existing local moments $\mu_{l}$. In contrast, in collinear Hartree-Fock calculations the increase of $\mu_{T}$ is associated to the formation of the local moments themselves since $\mu_{T}$ approximately proportional to $\mu_{l}$ [19]. Comparison with exact calculations shows that the present noncollinear picture is qualitatively closer to the actual ground-state magnetic properties than the one derived in collinear calculations, at least for the single-band Hubbard model. Indeed, we have computed the local moments $\mu_{l}^{2}=\left\langle S_{l}^{2}\right\rangle$ and the total spin $S$ of the same fcc 13 -atom cluster $(\nu=14)$ as a function of $U / t$ by using a Lanczos exact diagonalization method. Starting from the uncorrelated limit $\left[\mu_{1}^{2}(U=0)=0.28\right.$ and $\mu_{l}^{2}(U=0)=0.36$ for $l=2-$ 13)] $\mu_{l}^{2}$ increases rapidly with $U / t$ reaching values close to saturation already for $U / t \simeq 10\left[\mu_{1}^{2}(U=10)=0.56\right.$ and $\mu_{l}^{2}(U=10)=0.64$ while $\mu_{1}^{2}(U=\infty)=0.56$ and $\left.\mu_{l}^{2}(U=\infty)=0.70(l=2-13)\right]$. In contrast the groundstate spin $S$ remains equal to zero up to $U / t \simeq 40$. This implies that for $\nu=N+1=14$ the local moments are formed well before FM order sets in, as observed in the noncollinear UHF calculations (Fig. 5). Notice, however, that the situation could be different for other band fillings where ground-state ferromagnetism is found at much smaller $U / t$ or where $S$ is a non-monotonous function of $U / t$ (e.g., for $\nu=15-18$ [5]). UHF yields larger local magnetic moments at the cluster surface in agreement with the exact results but it underestimates severely both the Coulomb repulsion $U / t$ above which $\mu_{T}>1 / 2$ and the $U / t$ for reaching saturation.

Calculations have been also performed for fcc-like 13atom clusters having $\nu=N-1=12$ electrons. For small $U(U / t<4.9)$ the calculated magnetic order is collinear $\left(\left\langle\vec{S}_{l}\right\rangle \| \hat{z}\right)$. The UHF ground state is a broken symmetry state that shows a charge- and spin-density wave along the (001) direction. For $U / t<3.5$ the atoms $l$ in the central (001) plane present an enhanced electron density $\left\langle n_{l}\right\rangle \simeq 1.12$ and local magnetic moments $\mu_{l}=0$. The atoms of the upper and lower (001) planes show AF order within each plane and $\mu_{l}=0.17$. The vanishing $\mu_{l}$ at the central (001) plane can be interpreted as a consequence of magnetic frustrations since the sum of their NN spins is zero. For $U / t>3.5$, the surface atoms at the central (001) plane develop local moments $\mu_{l} \neq 0$. The spin arrangement remains collinear with important frustrations at some triangular faces. For $U / t>4.9$ the spin-density distribution changes to noncollinear with all surface spin having the same modulus. However, the central site remains unpolarized. In fact the magnetic order is the same as the one obtained if the central site is removed $(N=12)$. The moments at the triangular faces point along the medians just as in the classical antiferromagnetic ground state of an isolated triangle 11. In spite of the three-dimensional geometry of the cluster the spin structure is two-dimensional (all magnetic moments lie on the same plane).

The topology and symmetry of the cluster structure plays a major role in determining the magnetic order and magnetic correlations within the cluster. In this context, it is interesting to consider different geometries and to compare their magnetic behavior. In Fig. 6 the UHF magnetic order in a 13-atom icosahedral cluster having $\nu=12$ electrons is illustrated for representative values of $U / t$. As a result of degeneracies in the single-particle spectrum $(U=0)$ a noncollinear, coplanar arrangement of the local magnetic moments is found at the surface already for very small $U / t(U / t<5$, see Fig. 6). At the central atom (not shown in the figure) the magnetic moment $\mu_{1}=0$. A similar behavior is observed in fcc clusters $(N=13, \nu=12)$. The tendency to avoid frustrations within NN triangular rings conditions the arrangement of the local moments. In some triangles the spin polarizations point along the medians just as in the classical Heisenberg model, while in others two spins are parallel (i.e., fully frustrated). For larger values of $U / t$ $(U / t>5)$ a less frustrated solution is favored by the an- 
tiferromagnetic correlations. This corresponds to a truly three-dimensional arrangement of the surface moments with $\mu_{1}=0$. The spin structure is such that if the surface $\left\langle\vec{S}_{l}\right\rangle$ are brought to a common origin they form an icosahedron. The magnetic moments $\left\langle\vec{S}_{l}\right\rangle$ on pentagonal rings present a small component that is perpendicular to the plane containing the atoms and that is antiparallel to the magnetic moment of the atom capping the ring. The projections of $\left\langle\vec{S}_{l}\right\rangle$ on to the plane of the ring are ordered in the same way as in an isolated pentagon.

\section{COMPARISON BETWEEN UHF AND EXACT RESULTS}

In Fig. 17 UHF and exact results are given for the local moments $\mu_{l}^{2}=\left\langle S_{l}^{2}\right\rangle$ and spin correlations $\left\langle\vec{S}_{l} \cdot \vec{S}_{m}\right\rangle$ in an fcc-like 13-atom cluster. The UHF results for $\mu_{l}^{2}$ are quantitatively not far from the exact results. Not only the uncorrelated limit $(U=0)$ is reproduced, but good agreement is also obtained in the large $U / t$ regime. Main trends such as the larger $\mu_{l}^{2}$ at the cluster surface for small $U / t$ and the reduction of the difference between surface and inner moments for large $U / t$ are correctly given. However, UHF underestimates the increase of $\mu_{l}^{2}$ for $U / t<3.7$ and anticipates the tendency to localization with increasing $U / t$. This results in a much more rapid crossover from weak to strong interacting regimes than in the exact solution. The quantitative differences are more important in the case of spin correlation functions $\left\langle\vec{S}_{l} \cdot \vec{S}_{m}\right\rangle$ particularly for large $U / t$ [Fig. /(b)]. Here we find that UHF underestimates the strength of AF spin correlations $\gamma_{11}$ at the surface, since the formation of permanent local moments blocks quantum spin fluctuations along the transversal directions. Still, in both UHF and exact calculations, the increase of AF correlations at the surface (increase of $\left|\gamma_{11}\right|$ ) is done at the expense of a decrease of the spin correlations with the central atom (decrease of $\left|\gamma_{01}\right|$ ). The discrepancies between UHF and exact results for $N=13$ show the limits of mean-field and give us an approximate idea of the corrections to be expected in correlated calculations on larger clusters. As expected, UHF yields better results for properties like the local moments, that are related to the density distribution, than for the correlation functions. Nevertheless, since the trends given by UHF are qualitatively correct, it is reasonable to expect that the conclusions derived for larger clusters are also valid (Figs. 1 1 and 1 ).

The determination of the structure of magnetic clusters is a problem of considerable importance since structure and magnetic behavior are interrelated [2 5]. Moreover, since most calculations of cluster structures are based upon mean field approximations, it would be very interesting to evaluate the role of electron correlations. We have therefore determined the relative stability of a few representative cluster structures as a function of band filling $\nu / N$ and Coulomb repulsion strength $U / t$ in the framework of the UHF approximation to the Hubbard model and we have compared the results with available exact calculations [57. Four different symmetries are considered: icosahedral clusters, which maximize the average coordination number, face centered cubic (fcc) and hexagonal close packed (hcp) clusters, as examples of compact structures which are found in the solid state, and body centered cubic (bcc) clusters, as an example of a rather open bipartite structure. These cluster geometries are representative of the various types of structures found to be the most stable in rigorous geometry optimizations for $N \leq 8$ 晒. The results for $N=13$ are summarized in the form of a magnetic and structural diagram shown in Fig. 8. A qualitative description of the type of magnetic order obtained in the self-consistent calculations is indicated by the different shadings. One may distinguish three different collinear spin arrangements: non-magnetic solutions (NM), non-saturated or weak ferromagnetic solutions (WFM) and saturated ferromagnetic solutions (SFM). The NM case includes paramagnetic states in which the total moment $\mu_{T}$ is minimal $\left(\mu_{T}=0\right.$ or $\left.1 / 2\right)$. Concerning the noncollinear spin arrangements we distinguish two cases: noncollinear nonmagnetic states (NC) in which non-vanishing (eventually large) local moments $\mu_{l}$ sum up to an approximately minimal total moment $\mu_{T}<1$, and noncollinear ferromagnetic states (NCFM) that show a net magnetization $\mu_{T} \geq 1$. The NC states include all sort of frustrated antiferromagnetic-like spin structures, for example, those illustrated in Figs. 2 and 6. In order to quantify the effect of electron correlations we also show in Fig. 9 the corresponding magnetic and structural diagram as recently obtained by using exact diagonalization methods [5].

For small $U / t(U / t<10)$ the UHF results for the most stable of the considered structures are in very good agreement with the exact calculations [compare Figs. 8 and 9 . The icosahedral cluster yields the lowest energy in the low carrier-concentration regime $(\nu \leq 6)$, as could have been expected taking into account that the largest coordination number is favored for small $\nu$ as in smaller clusters [1]. In this case, the kinetic energy dominates (uncorrelated limit) and therefore the structure with the largest bandwidth is stabilized. As $\nu$ is increased several structural transitions occur. For small $U / t$, both exact and UHF calculations present the same structural changes: from icosahedral to fcc structure at $\nu=11$, from fcc to hcp at $\nu=17$, and from hcp to bcc at $\nu=20$. At larger $U / t(U / t>12)$ the interplay between the kinetic and Coulomb energies introduces important correlations that cannot be accounted for within UHF and that play a central role in the determination of the magnetic and structural properties. Thus, UHF often fails to yield the lowest-energy structure in the limit of large $U / t$. A main source of discrepancy is the too strong tendency of UHF to yield SFM ground states, particularly above half-band filling, which often disagrees with the exact magnetic behavior. Consequently the optimal structure is missed rather frequently. For example, for $\nu=19$ and large 
$U / t$, UHF predicts the fcc structure with a SFM ground state, while in the exact calculation the icosahedral cluster with $S=1 / 2$ is the optimum. Similar drawbacks are seen for other band fillings such as $\nu=7,10,21$, and 22 (large $U / t$ ). Still, in the event that the true ground state does show strong ferromagnetism, UHF succeeds since the ground state is the superposition of single-particle states. Examples of this kind are $\nu=12$ and $\nu=20$ for large $U / t$. These are rather exceptions, however, since the presence of a FM ground state in the exact solution is in general much less frequent than predicted by UHF. Around half-band filling UHF reproduces qualitatively well the transition from fcc or hcp to icosahedral structure with increasing $U / t$ as well as nontrivial re-entrant effects $(\nu=13-18)$. However, UHF underestimates the value of $U / t$ at which the structural changes occur (see Figs. 8 and 9). Part of the quantitative differences could be remedied by using in the UHF calculations a reduced or renormalized $U / t$ which simulates some of the effects of correlations.

Summarizing, UHF fails to reproduce the exact phase diagram in detail, particularly in some of the most interesting antiferromagnetic or weak ferromagnetic regimes. Structural transition are sometimes missing (e.g., for $\nu=10)$ and in other cases changes of structures appear artificially (e.g., bcc to hcp for $\nu=21$ and 22). Nevertheless, it is also fair to say that UHF yields a good account of the relative stability between the considered structures well beyond the weakly interacting limit (up to $U / t \simeq 16$ ) and that it also explains the larger stability of ferromagnetism above half-band filling. In the limit of strong interactions the validity of UHF breaks down and correlation effects beyond the single-determinant wavefunction are indispensable in order to obtain the groundstate structure and magnetic behavior reliably. Improvements on the UHF wave function could be introduced by restoring the broken spin symmetry as proposed in Refs. [14,15] [Eqs. (2.10 2.12)]. However, the success of such an approach is likely to depend on the geometry of the cluster. For example, in low symmetry structures UHF tends to exaggerate the formation of spin and charge density redistributions which may be far from the actual exact solution and which can be very difficult to restore $a$ posteriori. In such cases a Jastrow-like variational ansatz on restricted Hartree-Fock states could be more appropriate. Finally, it should be recalled that the Hubbard model for small clusters with one orbital per site is probably the most extremely low-dimensional system one may consider. Therefore, fluctuations and correlations effects are expected to be here more drastic than in larger clusters or in realistic multiband models more appropriate for the description of TM's.

\section{ACKNOWLEDGMENTS}

This work was supported by CONACyT (Mexico) and CNRS (France). One of the authors (MAO) acknowledges scholarships from CONACyT and FAI (UASLP).

[1] See, for instance, Proceedings of the 8th International Symposium on Small Particles and Inorganic Clusters (ISSPIC), Cophenague, Denmark, July 1-6, 1996, H. H. Andersen, Ed., Springer Physics Vol. XVI (1997) and Proceedings of ISSPIC-9, Lausanne, Switzerland, 1-5, 1998, Eur. Phys. J. D (1999), in press.

[2] D.R. Salahub and R.P. Messmer, Surf. Sci. 106, 415 (1981); C.Y. Yang, K.H. Johnson, D.R. Salahub, J. Kaspar and R.P. Messmer, Phys. Rev. B 24, 5673 (1981); K. Lee, J. Callaway and S. Dhar, Phys. Rev. B 30, 1724 (1985); K. Lee, J. Callaway, K. Wong, R. Tang and A. Ziegler, ibid. 31, 1796 (1985); K. Lee and J. Callaway, ibid. 48, 15358 (1993); G.M. Pastor, J. Dorantes-Dávila and K.H. Bennemann, Physica B 149, 22 (1988); Phys. Rev. B 40, 7642 (1989); B.I. Dunlap, Z. Phys. D 19, 255 (1991); J.L. Chen, C.S. Wang, K.A. Jackson and M.R. Perderson, Phys. Rev. B 44, 6558 (1991); M. Castro and D.R. Salahub, Phys. Rev. B 49, 11842 (1994); P. Ballone and R.O. Jones, Chem. Phys. Lett. 233, 632 (1995); B.V. Reddy, S.N. Khanna and B.I. Dunlap, Phys. Rev. Lett. 70, 3323 (1993); B. Piveteau, M-C. Desjonquéres, A. M. Olés and D. Spanjaard, Phys. Rev. B 53, 9251 (1996); J. Dorantes-Dávila, P. Villaseñor-González, H. Dreyssé and G.M. Pastor, ibid. 55, 15084 (1997).

[3] L. M. Falicov and R.H. Victora, Phys. Rev. B 30, 1695 (1984); Y. Ishii and S. Sugano, J. Phys. Soc. Jpn. 53, 3895 (1984); J. Callaway, D.P. Chen and R. Tang, Z. Phys D 3, 91 (1986); Phys. Rev. B 35, 3705 (1987).

[4] G.M. Pastor, R. Hirsch, and B. Mühlschlegel, Phys. Rev. Lett. 72, 3879 (1994); Phys. Rev. B 53, 10382 (1996).

[5] F. López-Urías and G.M. Pastor, Phys. Rev. B (1999), in press.

[6] D. R. Penn, Phys. Rev. 142, 350 (1966)

[7] F. Keffer, in Encyclopedia of Physics, vol. 18/2, edited by H.P.J. Wijn (Springer, Berlin, 1966); Y. Tsunoda, J. Phys. Condens. Matter 1, 10427 (1989).

[8] M. V. You, and V. Heine, J. of Phys. F 12, 177 (1982).

[9] R. Lorenz, J. Hafner, S. S. Jaswal and D. J. Sellmyer, Phys. Rev. Lett., 74, 3688 (1995); A. V. Smirnov and A. M. Bratkovsky, Europhys. Lett. 33, 527 (1996); L.M. Sandratskii, and J. Kübler, Phys. Rev. Lett. 76, 4963 (1996); L. Nordström and D. J. Singh, Phys. Rev. Lett. 76, 4420 (1996).

[10] D. Coffey and S. A. Trugman, Phys. Rev. Lett. 69, 176 (1992); F. Willaime and L. M. Falicov, J. Chem. Phys. 98, 6369 (1993); L. Bergomi, J. P. Blaizot, Th. Jolicoeur, and E. Dagotto, Phys. Rev. B 47, 5539 (1993).

[11] M. A. Ojeda-López, J. Dorantes-Dávila and G.M. Pastor, J. Appl. Phys. 81, 4170 (1997). 
[12] T. Oda, A. Pasquarello, and R. Car, Phys. Rev. Lett. 80, 3622 (1998).

[13] J. Hubbard, Proc. R. Soc. London, A 276, 238 (1963); ibid A281, 401 (1964); J. Kanamori, Prog. Theor. Phys. 30, 275 (1963); M. C. Gutzwiller, Phys. Rev. Lett. 10, 159 (1963).

[14] L. M. Falicov and R. A. Harris, J. Chem. Phys. 51, 3153 (1969).

[15] S. L. Reindl and G. M. Pastor, Phys. Rev. B 47, 4680 (1993).

[16] C. Lanczos, J. Res. Nat. Bur. Stand. 45, 255 (1950); B.N. Parlett, The Symmetric Eigenvalue Problem, (PrenticeHall, Engelwood Cliffs, 1980); J.K. Collum and R.A. Willoughby, Lanczos Algorithms for Large Symmetric Eigenvalue Computations, (Boston, 1985), Vol. I.

[17] In the present model without spin-orbit interactions the energy is invariant after any rotation of the complete spin arrangement with respect to the cluster structure. Only the relative orientations of the $\left\langle\vec{S}_{l}\right\rangle$ are significant. The choice made in the figures is arbitrary and obeys only illustrative purposes.

[18] Y. Nagaoka, Solid State Commun. 3, 409 (1965); D. J. Thouless, Proc. Phys. Soc. London 86, 893 (1965); Y. Nagaoka, Phys. Rev. 147, 392 (1966); H. Tasaki, Phys. Rev. B 40, 9192 (1989).

[19] J. Dorantes-Dávila, H. Dreyssé and G.M. Pastor, Phys. Rev. B 46, 10432 (1992). 


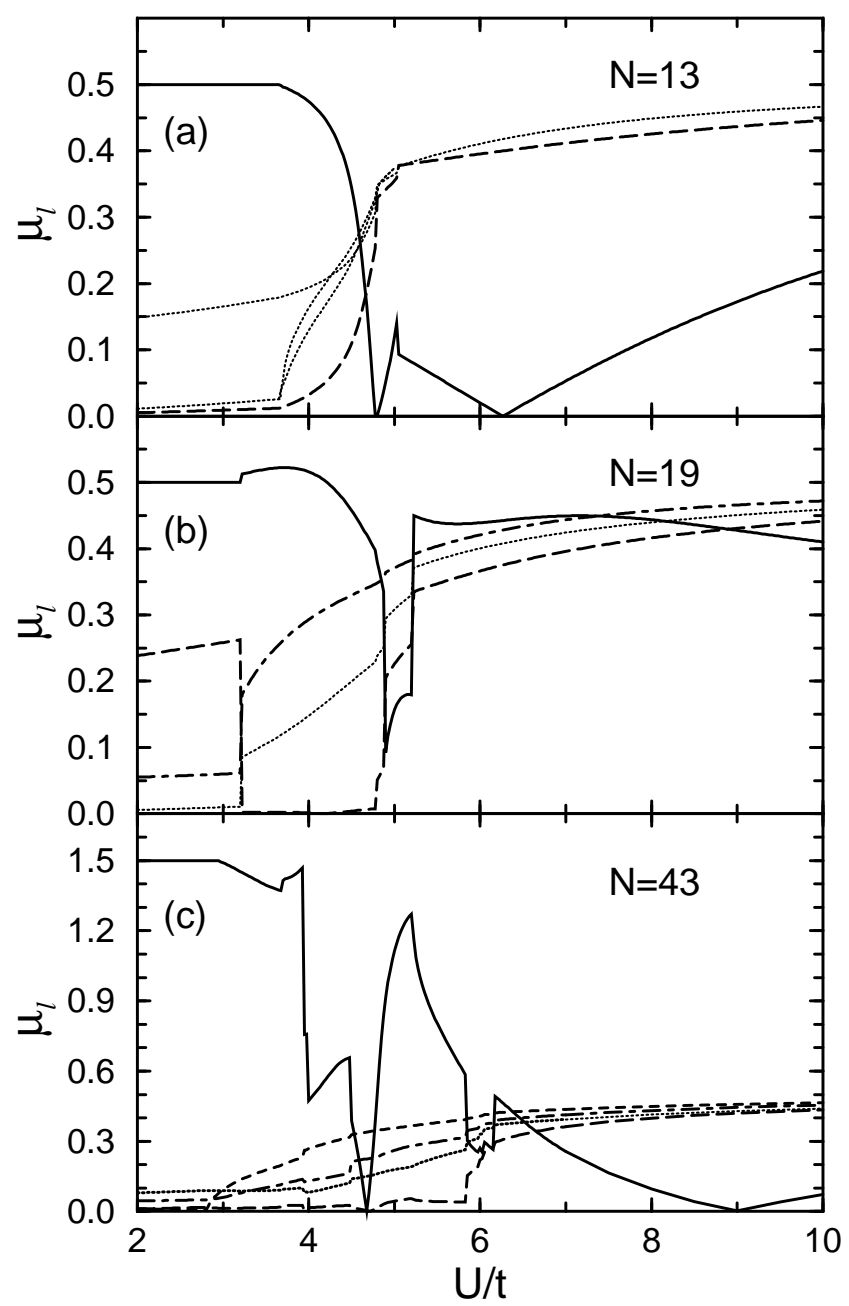

FIG. 1. Magnetic moments as a function of the Coulomb repulsion strength $U / t$ for fcc-like clusters having (a) $N=13$, (b) $N=19$ and (c) $N=43$ atoms at half-band filling (UHF approximation). Local moments $\mu_{l}=\left|\left\langle\vec{S}_{l}\right\rangle\right|$ are shown for the central atom $l=1$ (dashed), its first NN's $(l=2-13$, dotted), second NN's $(l=14-19$, dashed-dotted $)$ and third NN's ( $l=20-43$, short dashed). In (b) and (c) $\mu_{l}$ refers to the shell average. The total moment $\mu_{T}=\left|\sum_{l}\left\langle\vec{S}_{l}\right\rangle\right|$ is given by the solid curves. 

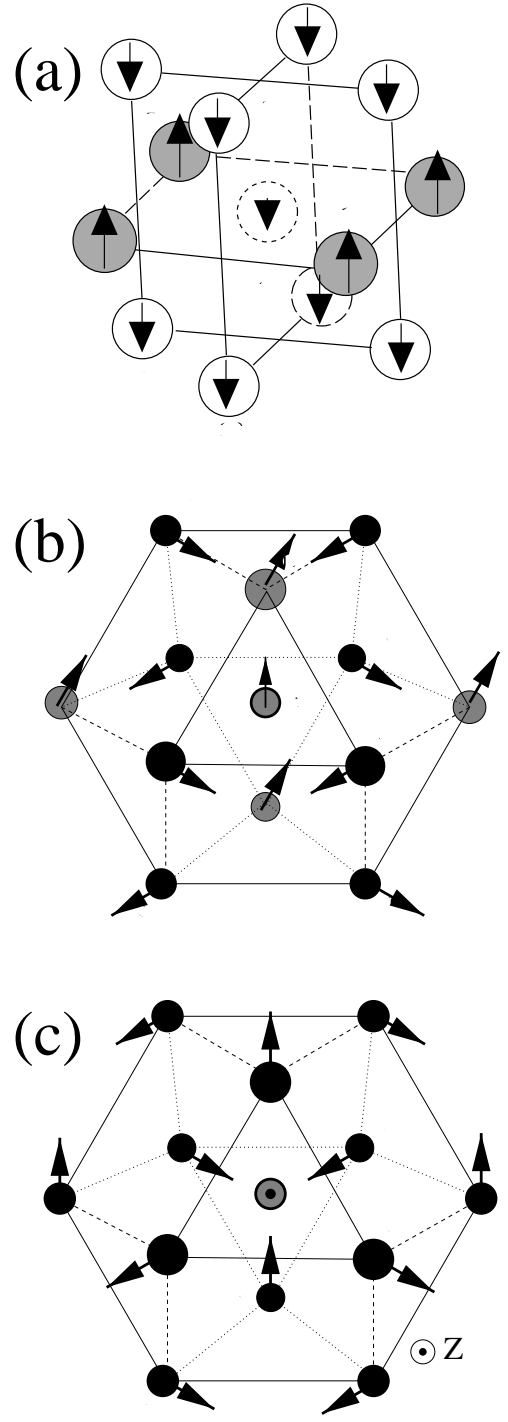

FIG. 2. Illustration of the UHF magnetic order in fcc-like clusters having $N=13$ atoms, $\nu=N=13$ electrons and representative values of $U / t$ (Hubbard model) [17]. (a) $U / t<3.7$ : The surface atoms in grey have larger local magnetic moments $\left|\left\langle\vec{S}_{l}\right\rangle\right|=0.16-0.18$ and less electron density $\left\langle n_{l}\right\rangle \simeq 0.88-0.90$ than the other surface atoms for which $\left|\left\langle\vec{S}_{l}\right\rangle\right| \simeq 0.02-0.03$ and $\left\langle n_{l}\right\rangle=1.05-1.12$. The spin arrangement is collinear. (b) $4.8 \leq U / t<5.1$ : The plane of the figure is perpendicular to the (111) direction. The arrows show the local spin polarizations $\left\langle\vec{S}_{l}\right\rangle$ which are all within the (111) plane. (c) $U / t \geq 5.1$ : The plane of the figure is perpendicular to the (111) direction. The arrows show the projection of $\left\langle\vec{S}_{l}\right\rangle$ on to the (111) plane. At the central atom $\left\langle\vec{S}_{1}\right\rangle \|(111)$. The off-plane components of $\left\langle\vec{S}_{l}\right\rangle$ at the surface atoms are antiparallel to the central spin and have all the same value $\left(\left|\left\langle S_{l}^{z}\right\rangle\right| \simeq 0.023-0.056\right.$ for $l=2-13)$.

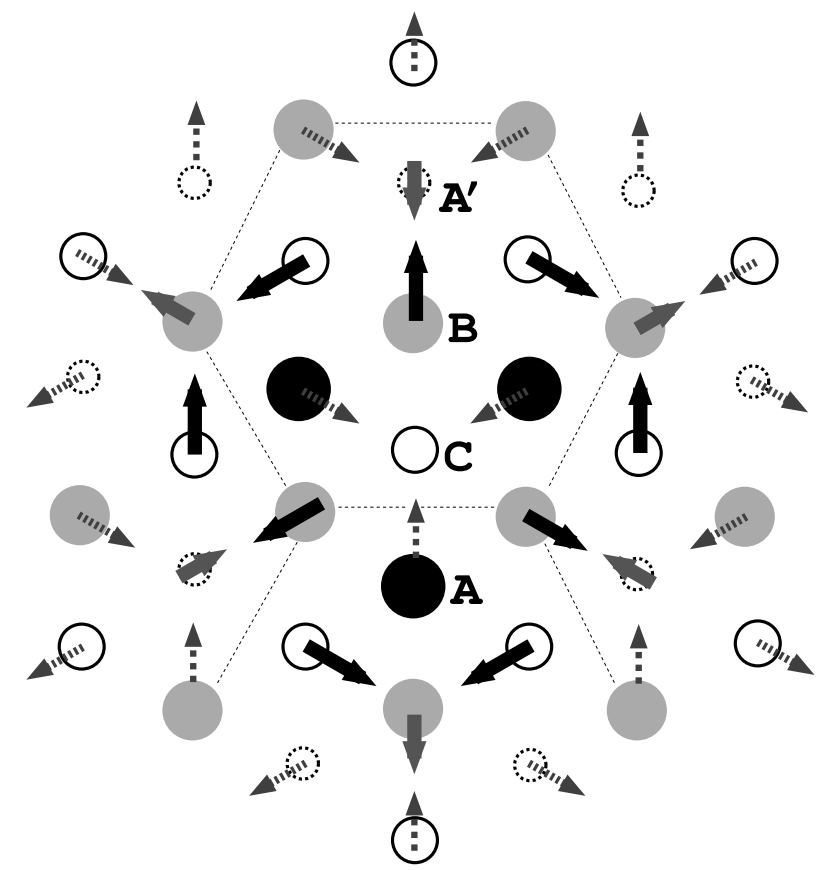

FIG. 3. Illustration of the local magnetic moments $\left\langle\vec{S}_{l}\right\rangle$ in a 43-atom fcc-like cluster at half-band filling and $U / t=10$ [17]. As in Fig. 2(c), the plane of the figure is perpendicular to the (111) direction. The atoms are represented by circles using different sizes and grey tones for different (111) layers. The later are denoted by A, B, C and A' starting from above. The arrows show the projection of $\left\langle\vec{S}_{l}\right\rangle$ on to the (111) plane. At the central atom $\left\langle\vec{S}_{1}\right\rangle \|(111)$. The spin structure is 3-dimensional with off-plane components of $\left\langle\vec{S}_{l}\right\rangle$ that alternate sign on different shells around the central atom. For the 13 innermost atoms the magnetic order is similar to the one shown in Fig. B(c). 


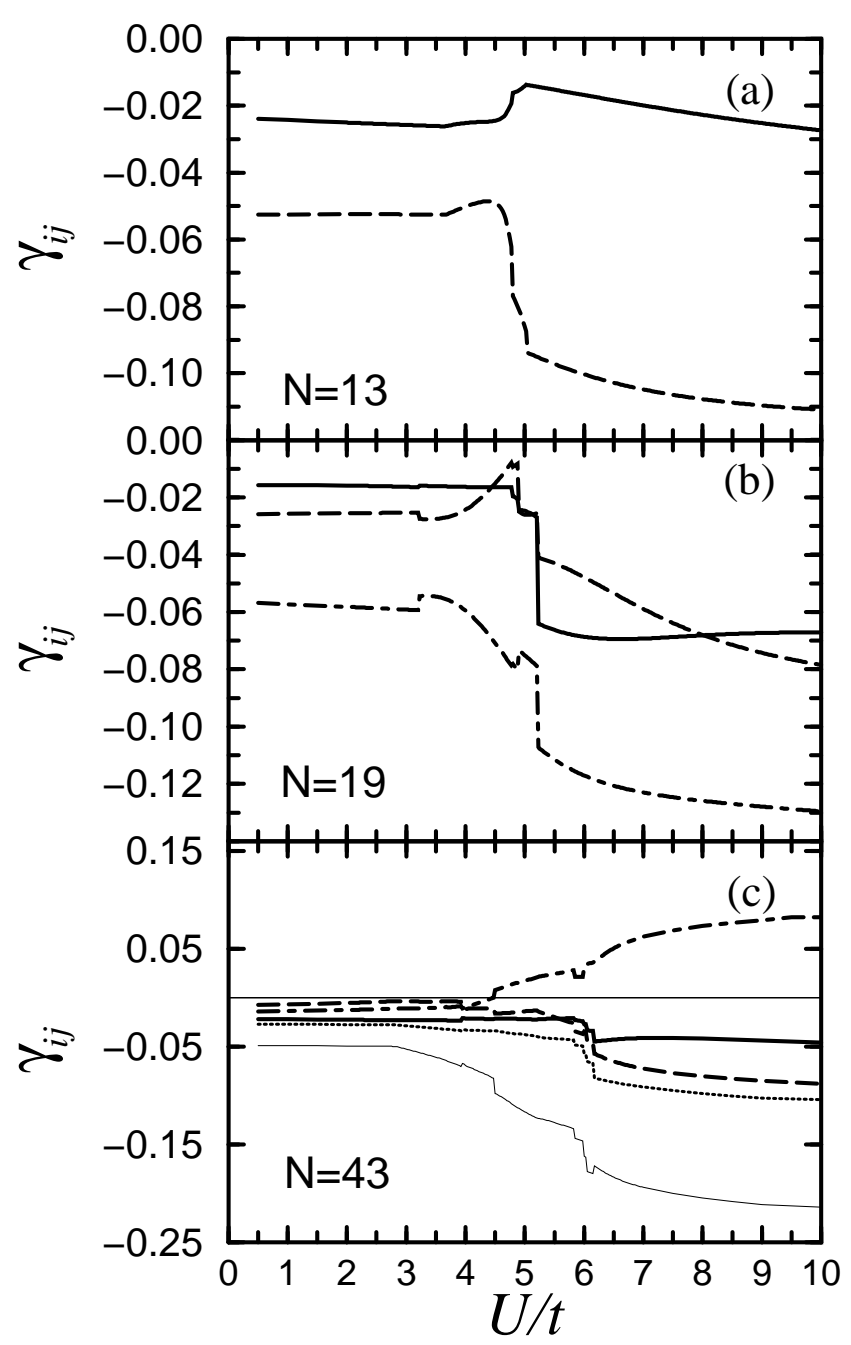

FIG. 4. Spin correlations $\left\langle\vec{S}_{l} \cdot \vec{S}_{m}\right\rangle$ in fcc-like clusters having $N=13-43$ atoms as a function of $U / t$. Results are given for the average spin correlations $\gamma_{01}$ between the central site and its first NN's (solid), $\gamma_{11}$ between NN sites on the first shell (dashed), $\gamma_{12}$ between NN sites on the first and second shell (dashed-dotted), $\gamma_{13}$ between NN sites on the first and third shell (dotted), and $\gamma_{23}$ between NN sites on the second and third shell [lower thin curve in (c)].

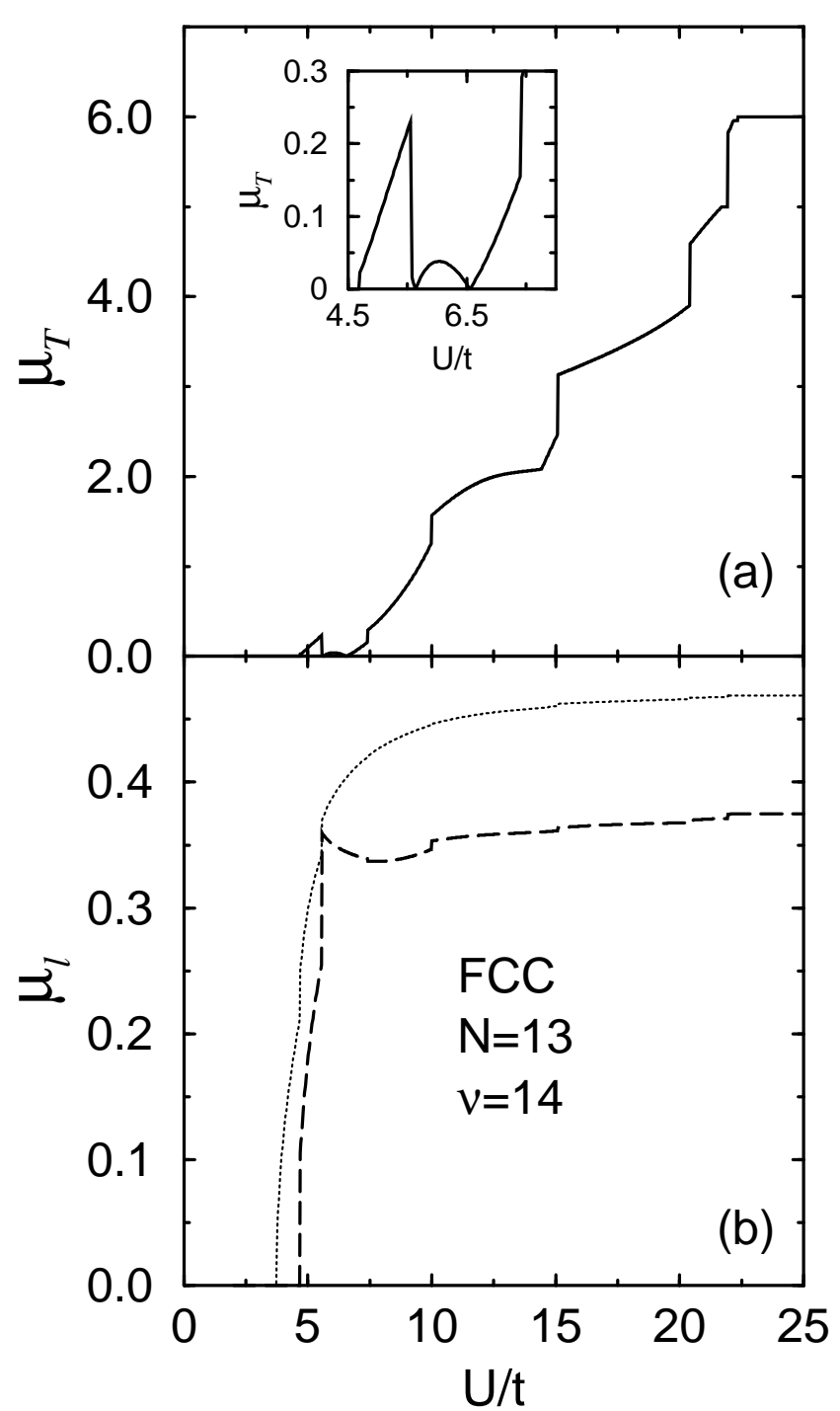

FIG. 5. Magnetic moments in an fcc-like 13-atom cluster with $\nu=14$ electrons as a function of $U / t$ (UHF approximation): (a) total moment $\mu_{T}=\left|\sum_{l}\left\langle\vec{S}_{l}\right\rangle\right|$ and (b) local moments $\mu_{l}=\left|\left\langle\vec{S}_{l}\right\rangle\right|$ at the central site (dashed line) and at the surface (dotted line). 


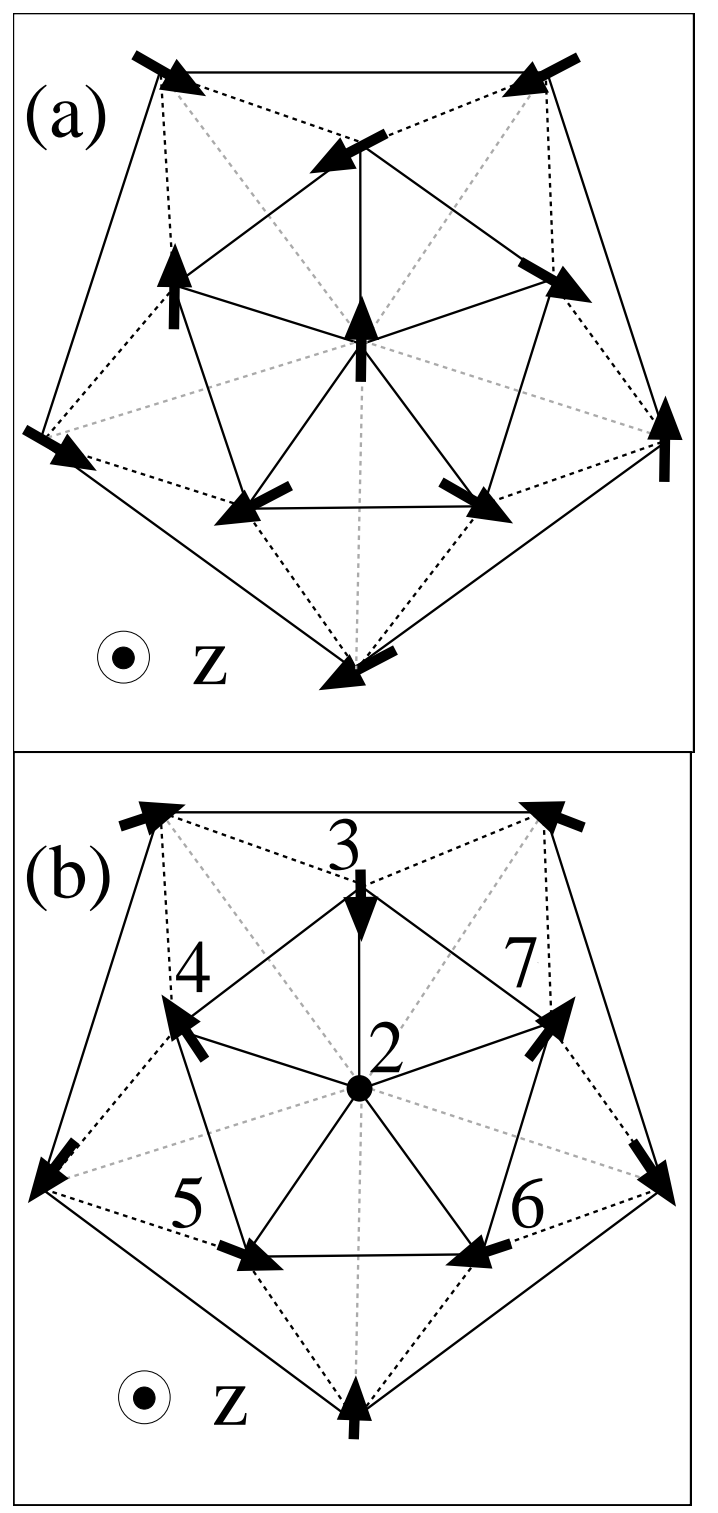

FIG. 6. Illustration of the magnetic order at the surface of an icosahedral 13-atom cluster with $\nu=N-1=12$ electrons (UHF approximation) [17. (a) $0.8<U / t \leq 5$ : All local magnetic moments $\left\langle\vec{S}_{l}\right\rangle$, represented by the arrows, are parallel to the plane of the figure. (b) $U / t>5$ : The arrows show the projections of $\left\langle\vec{S}_{l}\right\rangle$ on the plane of the figure. At site $l=2,\left\langle\vec{S}_{l}\right\rangle$ is perpendicular to the plane of the figure (along the $z$ axis). The $\left\langle\vec{S}_{l}\right\rangle$ at sites $l=3-7$ have antiparallel projection to $\left\langle\vec{S}_{2}\right\rangle\left[\cos \theta_{2 l}=\left(\left\langle\vec{S}_{l}\right\rangle \cdot\left\langle\vec{S}_{2}\right\rangle\right) /\left(\left|\left\langle\vec{S}_{l}\right\rangle\right|\left|\left\langle\vec{S}_{2}\right\rangle\right| \simeq-0.45\right)\right]$. If brought to a common origin, the spin polarizations $\left\langle\vec{S}_{l}\right\rangle$ form a perfect icosahedron. Notice that the positions of the atoms in the lower pentagonal rings have been expanded along the polar radius in order to ease the visualization.

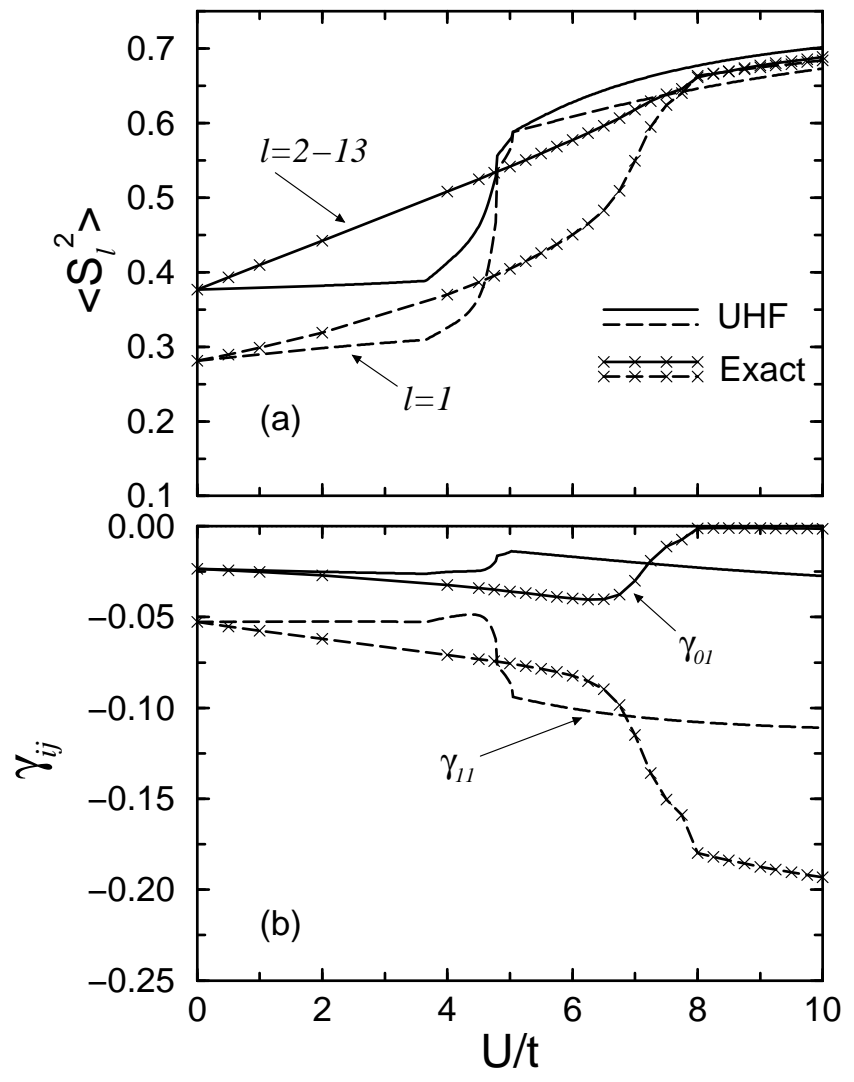

FIG. 7. Comparison between UHF and exact results for an fcc-like 13-atom cluster at half-band filling. (a) Local magnetic moments $\mu_{l}^{2}=\left|\left\langle\vec{S}_{l} \cdot \vec{S}_{l}\right\rangle\right|$ at the central site $(l=1$, dashed curves) and at the cluster surface $(l=2-13$, solid curves $)$. (b) Average $\gamma_{i j}$ of the spin correlation functions $\left\langle\vec{S}_{l} \cdot \vec{S}_{m}\right\rangle$ between the central site and its first NN's at the surface $\left(\gamma_{01}\right.$, dashed curves) and between NN's at the surface shell $\left(\gamma_{11}\right.$, solid curves). The curves with (without) crosses correspond to exact (UHF) results. 


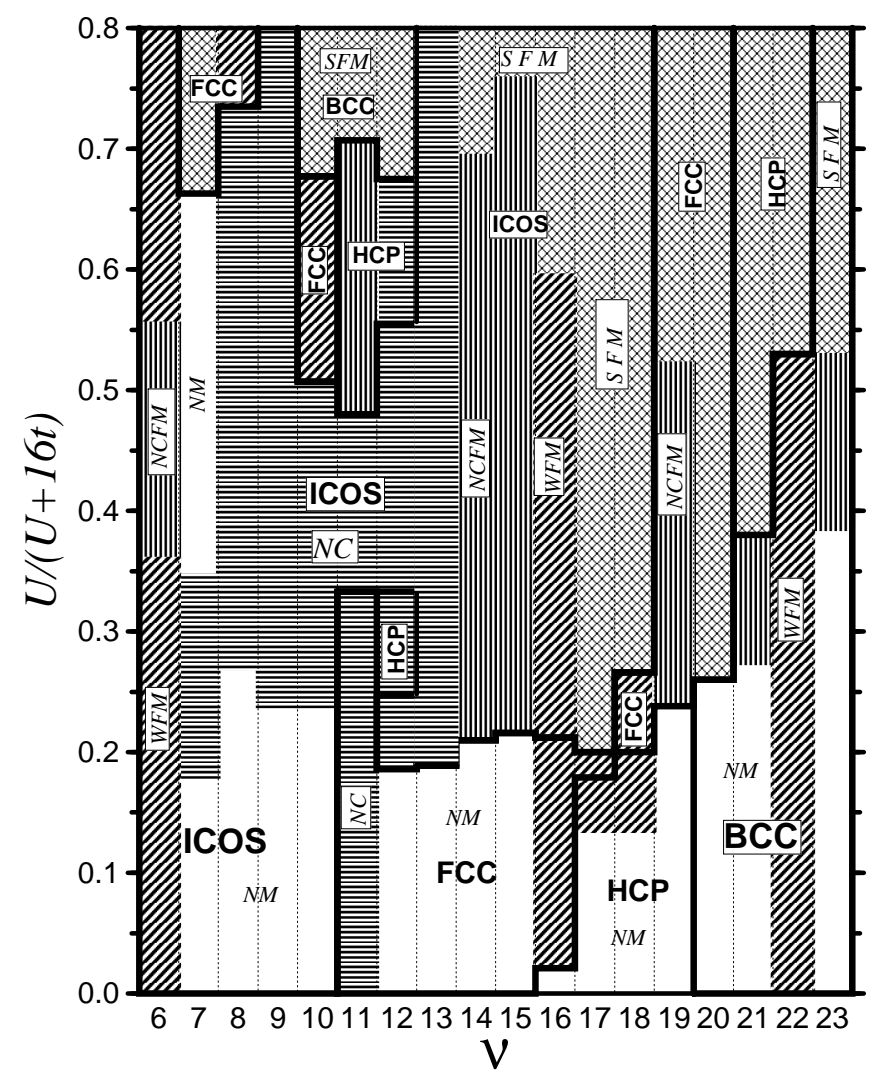

FIG. 8. Magnetic and structural diagram of clusters having $N=13$ atoms as obtained by using the Hubbard model in the unrestricted Hartree-Fock (UHF) approximation. Four different types of structures are considered: icosahedral (ICOS), face centered cubic (FCC), hexagonal close packed (HCP), and body centered cubic (BCC). The lowest-energy structure is given as a function of the Coulomb repulsion strength $U$, hopping integral $t$ and number of electrons $\nu$. The bold lines indicate the structural transitions. For $\nu<6$ the lowest-energy structure is icosahedral and for $\nu>23$ it is bcc. The corresponding UHF magnetic orders are indicated by different shading patterns: saturated ferromagnetic (crossed lines, $S F M$ ), collinear weak ferromagnetic (diagonal lines, $W F M$ ), collinear nonmagnetic (no shading, $N M$ ), noncollinear with total moment $\mu_{T}<1$ (horizontal lines, $N C$ ), and noncollinear with total moment $\mu_{T} \geq 1$ (vertical lines, NCFM).

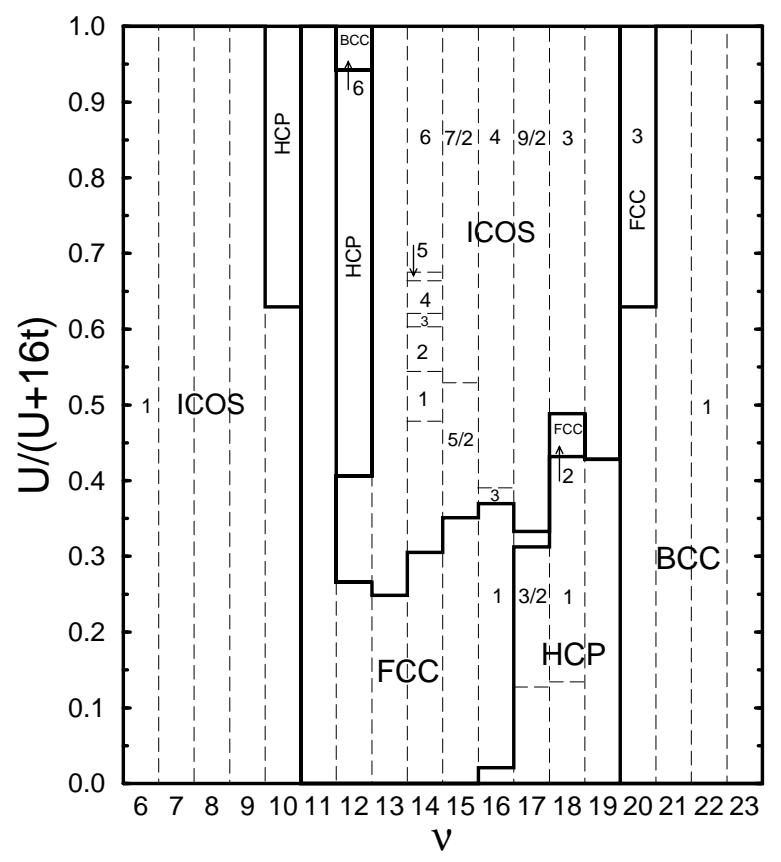

FIG. 9. Magnetic and structural diagram of Hubbard clusters having $N=13$ atoms as obtained by using exact diagonalization methods (after Ref. [7). As in Fig. 8, icosahedral (ICOS), face centered cubic (FCC), hexagonal close packed (HCP) and body centered cubic (BCC) structures are considered. The most stable structure is given as a function of Coulomb repulsion $U$, hopping integral $t$ and number of electrons $\nu$. The corresponding ground-state spin $S$ is minimal $(S=0$ or $1 / 2)$ unless explicitly indicated. Broken lines separate regions having the same structure but different $S$. For $\nu \leq 9$ the icosahedron yields the lowest energy for all $U / t$, while for $\nu \geq 21$ the bcc structure is the most stable. 